\title{
Densidade de estocagem e frequência alimentar no cultivo de jundiá em tanques-rede
}

\author{
Suziane Ghedini Martinelli(1), João Radünz Neto(1), Leila Picolli da Silva(1), Giovani Taffarel Bergamin (2), \\ Daniel Maschio(1), Marco Aurélio Lopes Della Flora(1), Lucas Mesquita da Costa Nunes ${ }^{(1)}$ e Glauber Possani( ${ }^{(1)}$ \\ (1)Universidade Federal de Santa Maria, Centro de Ciências Rurais, Departamento de Zootecnia, Avenida Roraima, no 1.000, \\ Cidade Universitária, Camobi, CEP 97105-900 Santa Maria, RS. E-mail: suzimartinelli@yahoo.com.br, jradunzneto@gmail.com, \\ leilasliva@yahoo.com.br, dani.maschio@hotmail.com, marco@zootecnista.com.br, lucasnunes_big@hotmail.com, gobe.possani.88@gmail.com \\ (2)Embrapa Pesca e Aquicultura, Quadra 104 Sul, Avenida LO1, o 34, Conjunto 4, 1ํ e 2ㅇ Pavimentos, Plano Diretor Sul, CEP $77020-020$ Palmas, \\ TO. E-mail: giovani.bergamin@embrapa.br
}

Resumo - O objetivo deste trabalho foi avaliar o efeito de diferentes densidades de estocagem e frequências alimentares sobre a resposta zootécnica e metabólica, e sobre a composição de peixe inteiro e de filé de juvenis de jundiá (Rhamdia quelen) cultivados em tanques-rede durante 60 dias. Foram utilizados 1.200 juvenis de jundiá $(57,48 \pm 3,34 \mathrm{~g}$ e $17,86 \pm 0,36 \mathrm{~cm})$. Os peixes foram distribuídos em delineamento inteiramente casualizado, em arranjo fatorial $2 \times 2$, à densidade de 50 e 150 peixes $\mathrm{m}^{-3}$ e frequência alimentar de uma ou duas vezes ao dia. Tanto as densidades de estocagem quanto o manejo alimentar não influenciaram o desempenho nem os parâmetros metabólicos. No entanto, a interação entre a menor densidade e a maior frequência alimentar resultou em menor teor lipídico nos filés. Como ambos os fatores testados não interferiram no desempenho zootécnico, pode-se sugerir como melhor densidade a de 150 peixes $\mathrm{m}^{-3}$, com frequência de arraçoamento de uma vez ao dia.

Termos para indexação: Rhamdia quelen, manejo alimentar, parâmetros metabólicos, qualidade de pescado.

\section{Stocking density and feeding rate of South American catfish reared in net cages}

\begin{abstract}
The objective of this work was to evaluate the effect of different stocking densities and feeding frequencies on the zootechnical and metabolic response and on the whole fish and fillet composition of juvenile South American catfish (Rhamdia quelen) cultured in net cages for 60 days. One thousand and two hundred juvenile catfish $(57,48 \pm 3,34 \mathrm{~g}$ and $17,86 \pm 0,36 \mathrm{~cm})$ were used. Fish were distributed in a completely randomized design, with a $2 \times 2$ factorial arrangement, at 50 and $150 \mathrm{fish} \mathrm{m}^{-3}$ and feeding frequency once or twice a day. Both stocking density and feeding influenced neither the performance, nor the metabolic parameters. However, the interaction between the lowest density and the highest feeding frequency resulted in the lowest lipid content in the fillets. As both tested factors did not affect the growth performance, $150 \mathrm{fish} \mathrm{m}^{-3}$ can be suggested as the best density together with a once-a-day feeding frequency.
\end{abstract}

Index terms: Rhamdia quelen, feed management, metabolic parameters, fish quality.

\section{Introdução}

A aquicultura brasileira apresentou crescimento de $43,8 \%$, entre os anos de 2007 e 2009, aumento este superior aos observados para as principais espécies zootécnicas (Brasil, 2010). Verificou-se que a produção de pescado cultivado apresentou evolução média superior a $25 \%$ entre 2007 a 2009, o que foi alicerçado na exploração de espécies exóticas, como a tilápia-do-nilo (Oreochromis niloticus) e as carpas (Ctenopharyngodon idella, Cyprinus carpio, Hypophthalmichthys molitrix e Aristichthys nobilis) (Brasil, 2010).
Existem várias espécies nativas com grande potencial para a piscicultura, entre as quais se destaca o jundiá (Rhamdia quelen), especialmente na Região Sul do Brasil (Baldisserotto \& Gomes, 2005). Tal espécie apresenta ampla adaptabilidade aos sistemas de cultivo, favorecida pelo hábito alimentar onívoro, por aceitar rações artificiais e por ter boa eficiência alimentar. Quanto à qualidade como pescado, sua carne é de excelente sabor e não apresenta espinhas intramusculares, que são características desejadas pelo mercado consumidor (Fracalossi et al., 2004). Em razão destes aspectos, o jundiá é uma espécie 
com elevado potencial de criação para a piscicultura brasileira.

A criação em tanques-rede pode ser alternativa promissora para intensificar o cultivo do jundiá, especialmente ao se considerar a ocupação produtiva de reservatórios, que são construídos com a finalidade de irrigação (cultivo de arroz irrigado) ou de geração de energia elétrica. Além disso, deve-se ponderar que o cultivo em tanques-rede tem menor custo na implantação do que o convencional (60 a 70\% menor), com maior facilidade de observação do estado sanitário e de despescas (Furlaneto et al., 2006).

Fracalossi et al. (2004), em estudo sobre o potencial do jundiá para a aquicultura, em viveiros de terra, encontraram resultados satisfatórios para os parâmetros de crescimento desta espécie, em cultivo de 1,30 peixes $\mathrm{m}^{-2}$, com ganho de peso diário de até 1,97 g, similar ao encontrado em cultivos de tilápia. Porém, há carência de estudos com espécies nativas, em sistemas de tanques-rede, especialmente no que diz respeito à densidade de estocagem e à frequência alimentar (Almeida \& Nuñer, 2009). Essas informações são relevantes para formar pacotes tecnológicos que auxiliem na divulgação e exploração intensiva das espécies nativas, o que pode gerar amplo retorno econômico e social para o País.

A frequência alimentar pode contribuir para a redução da conversão alimentar e aumento do ganho de peso (Carneiro \& Mikos, 2005). Também pode contribuir para o estímulo do peixe pela procura do alimento em momentos pré-determinados, o que deve reduzir o desperdício de alimento, manter a qualidade da água e reduzir os custos de produção. Segundo Canton et al. (2007), normalmente, quando se alimentam juvenis de diferentes espécies de peixe mais de uma vez ao dia, o ganho de peso aumenta.

Outro fator determinante para o crescimento é a densidade de estocagem. Peixes criados a baixas densidades podem acumular mais gordura, em razão da maior oferta de ração e da menor competição por alimento (Lazzari et al., 2011). Porém, baixa densidade conduz a um subaproveitamento do espaço para a criação dos peixes (Piaia \& Baldisserotto, 2000). Em altas densidades, há maior chance de estresse e respectivos efeitos sobre o sistema imunológico (Salaro et al., 2003) e maior probabilidade de degradação da qualidade da água (Jobling, 1994). A melhor densidade varia conforme a espécie, tamanho dos exemplares e sistema de cultivo (Lazzari et al., 2011).

O objetivo deste trabalho foi avaliar o efeito de diferentes densidades de estocagem e frequências alimentares, sobre a resposta zootécnica e metabólica e a composição de peixe inteiro e filé de juvenis de jundiás (Rhamdia quelen), cultivados em tanques-rede, durante 60 dias.

\section{Material e Métodos}

O experimento foi realizado no Laboratório de Piscicultura, da Universidade Federal de Santa Maria, RS (à altitude de $95 \mathrm{~m}, 2^{\circ} 43^{\prime} \mathrm{S}, 53^{\circ} 42^{\prime} \mathrm{W}$ ). Utilizaramse 12 tanques-rede de $1 \mathrm{~m}^{-3}$, dispostos em linha, com $1 \mathrm{~m}$ de distância entre eles. Os tanques foram dispostos em viveiro de terra de 0,1 ha de lâmina d'água e $1,50 \mathrm{~m}$ de profundidade. O sistema não possuía renovação de água, porém era provido de aeração mecânica (aerador de pás).

Cada tanque-rede correspondia a uma unidade experimental. Juvenis de jundiá (1.200) adquiridos da Estação Experimental de Piscicultura, da Universidade Regional Integrada do Alto Uruguai e das Missões, com massa e comprimento médios iniciais de $57,48 \pm 3,34 \mathrm{~g}$ e $17,86 \pm 0,36 \mathrm{~cm}$, respectivamente, foram distribuídos nas unidades experimentais e submetidos a sete dias de adaptação, antes do início do experimento.

Os peixes foram distribuídos em delineamento inteiramente casualizado, em arranjo fatorial $2 \times 2$, em que a densidade de estocagem representou $\mathrm{o}$ fator 1 (50 e 150 peixes $\mathrm{m}^{-3}$, equivalentes a $2,8 \mathrm{e}$ $8,7 \mathrm{~kg} \mathrm{~m}^{-3}$, respectivamente), e a frequência alimentar representou o fator 2 (uma e duas vezes ao dia), em quatro tratamentos e três repetições. A duração total do experimento foi de 60 dias (março a maio de 2010).

Os animais receberam ração comercial extrusada com $28 \%$ de proteína bruta, à razão de 3\% da biomassa por dia. Aqueles que recebiam uma refeição diária eram alimentados de forma integral às $18 \mathrm{~h}$, e aqueles tratados com duas alimentações recebiam $50 \%$ às $08 \mathrm{~h}$, e o restante às $18 \mathrm{~h}$. A alimentação foi realizada com auxílio de comedouros perimetrais, confeccionados com sombrite, para evitar perda de ração para o meio.

A cada 30 dias de experimento, realizavam-se biometrias para a avaliação do crescimento e o ajuste da quantidade de alimento em todos os peixes. Após 15 dias de cada biometria, a quantidade de alimento foi ajustada, tendo-se pesado $30 \%$ da população. 
Os parâmetros físicos e químicos da água, como temperatura e oxigênio dissolvido, foram aferidos duas vezes ao dia, pela manhã $(8 \mathrm{~h})$ e à tarde $(18 \mathrm{~h})$, com auxílio de potenciômetro digital portátil e oxímetro digital, modelo 550A (YSI-Yellowsprings, OH, EUA). Semanalmente, foram realizadas as demais análises de qualidade da água, tais como: $\mathrm{pH}$, em pHmetro digital Servylab, modelo mPA-210 (Servylab, São Leopoldo, RS); alcalinidade e dureza total (Adad, 1982); amônia total (Verdouw et al., 1997) e transparência (disco de Secchi).

Ao final do período experimental, os peixes foram submetidos a jejum de 24 horas, anestesiados com eugenol (solução de óleo de cravo, a $50 \mathrm{mg} \mathrm{L}^{-1}$ ) (Cunha et al., 2010), e foram, então, pesados e medidos (Certificado de Apresentação para Apreciação Ética $n^{\circ}$ 006973/2009-41).

As medidas zootécnicas avaliadas foram as seguintes: massa inicial (PI); massa final (PF); ganho de peso (GP); ganho médio diário (GMD); comprimento total (CT); taxa de crescimento específico $(\mathrm{TCE})=(\ln \mathrm{PF}-\ln \mathrm{PI}) /$ dias $\times 100$; e fator de condição $(\mathrm{FC})=\left[\right.$ peso $\left./(\mathrm{CT})^{3} \times 100\right]$.

Dois peixes de cada tanque foram selecionados para a coleta de sangue, por punção da veia caudal, para análise de proteínas totais, triglicerídeos, colesterol total, urato e glicose, a partir de kits bioquímicos colorimétricos (Doles). Após a coleta de sangue, estes peixes foram abatidos por imersão em água e gelo (proporção1:1), filetados e eviscerados, e seu fígado e trato gastrointestinal (TGI) foram separados e pesados. O TGI foi medido, para o cálculo do rendimento de carcaça e índices digestivos. De cada tanque, dois animais foram separados para a análise da composição centesimal (nos filés) da proteína, umidade, cinzas conforme Cunniff (1995), e gordura (Bligh \& Dyer, 1959).

Foram avaliados os parâmetros relacionados à carcaça e ao filé, como rendimento de carcaça $(\mathrm{RC})=[($ massa eviscerada com cabeça e brânquias $)$ / (massa inteira) $\times 100$ ]; rendimento de filé $(\mathrm{RF})$ $=[($ massa dos filés $/$ massa inteira $) \times 100]$; índice digestivo somático $($ IDS $)=[$ (massa do trato intestinal / massa inteira) $\times 100)$ ]; índice hepatossomático (IHS) $=[($ massa do fígado $/$ massa inteira $) \times 100]$; índice de gordura visceral $(\mathrm{IGV})=[($ massa da gordura visceral $/$ massa inteira $\times 100$ ] e quociente intestinal
$(\mathrm{QI})=[$ (comprimento do trato intestinal / comprimento total)] (Veiverberg et al., 2008).

A avaliação da uniformidade dos lotes de peixe, em cada unidade experimental, foi feita de acordo com Furuya et al. (1998). Foram utilizadas as médias de massa de cada unidade experimental, e foi quantificado o número de indivíduos com massa dentro do intervalo correspondente a $20 \%$ acima e abaixo da média, conforme a equação Uniformidade $(\mathrm{U})=[(\mathrm{N} / \mathrm{Nt}) \times 100]$, em que: $\mathrm{U}$ é a uniformidade (\%); N é o número total de animais no tanque; Nt é o número total de animais com massa de $\pm 20 \%$, dentro da média de peso vivo, em cada unidade experimental.

Os dados foram submetidos ao teste de normalidade (Shapiro-Wilk) e à análise de variância. As médias foram comparadas, a $5 \%$ de probabilidade, pelo teste de Duncan. A análise de interação entre os fatores e os dados foi realizada pelo pacote estatístico SPSS 8.0.

\section{Resultados e Discussão}

As médias de temperatura da água (manhã e tarde), oxigênio dissolvido (manhã e tarde), alcalinidade, dureza, transparência e $\mathrm{pH}$, durante o período experimental, foram respectivamente de: $22,04 \pm 0,46^{\circ} \mathrm{C}$ (manhã), 23,12 $\pm 0,75^{\circ} \mathrm{C}$ (tarde); 7,22 $\pm 0,11 \mathrm{mg} \mathrm{L}^{-1}$ (manhã), 9,24 $\pm 0,34 \mathrm{mg} \mathrm{L}^{-1}$ (tarde); 34,5 $\pm 1,04 \mathrm{mg} \mathrm{L}^{-1} \mathrm{de}$ $\mathrm{CaCO}_{3}, 56,0 \pm 18,47 \mathrm{mg} \mathrm{L}^{-1}$ de $\mathrm{CaCO}_{3}, 19,33 \pm 2,33 \mathrm{~cm}$; e $7,01 \pm 0,11$.

Ao final de 60 dias experimentais, verificou-se que a densidade de estocagem (50 ou 150 peixes $\mathrm{m}^{-3}$ ), a frequência alimentar (uma ou duas vezes ao dia) e a interação entre elas, não alteraram os valores de massa final, ganho médio diário, ganho de massa, comprimento total, sobrevivência e taxa de crescimento específico dos jundiás (Tabela 1).

$\mathrm{O}$ crescimento dos peixes pode ser comprometido, quando eles são submetidos a baixas densidades, em razão do subaproveitamento do espaço disponível e baixa produtividade por área. No entanto, a densidade elevada pode ser motivo de estresse e mortalidade dos peixes, além de comprometer a qualidade da água (Jobling, 1994).

A densidade de estocagem não produziu efeito sobre o desempenho zootécnico dos jundiás. Corrêia et al. (2010) também não observaram efeitos da densidade sobre o desempenho zootécnico de juvenis de jundiás e carpa-húngara (Cyprinus carpio), cultivados em 
policultivo ( $75 \%$ jundiá, $25 \%$ carpa-húngara), em sistema de recirculação de água, a 57 e 143 peixes $\mathrm{m}^{-3}$, durante quatro semanas. Os autores afirmam que estes resultados são consequência do curto período experimental e das baixas densidades estabelecidas.

A frequência alimentar pode contribuir para a melhoria da produção e produtividade final do cultivo, com redução da conversão alimentar e melhorias no ganho de massa (Carneiro \& Mikos, 2005). No entanto, não foram observadas diferenças significativas no desempenho zootécnico do jundiá entre as frequências de arraçoamento testadas (Tabela 1). Ao testar frequências alimentares em jundiás, Carneiro \& Mikos (2005) não observaram diferenças quanto aos dados de crescimento. Isso significa que o manejo alimentar adotado pode não interferir na produtividade do jundiá.

A densidade de estocagem e a frequência de arraçoamento também não afetaram os rendimentos de carcaça (Tabela 1). Os dados corroboram os de Losekann et al. (2008), que obtiveram valores entre $80-87 \%$ de rendimento de carcaça, em jundiás alimentados com dietas com óleos de arroz, canola ou soja.

Os índices hepatossomático (IHS) e digestivossomático (IDS), assim como o quociente intestinal (QI) também não apresentaram diferenças entre os tratamentos (Tabela 2). Pedron et al. (2008) e Reidel et al. (2010) encontraram valores de IDS de 2,76 a 3,28 e IHS de 2,25 a 3,75, respectivamente, em jundiás cultivados em tanques-rede, e os resultados desses estudos são semelhantes aos encontrados no presente trabalho. Corrêia et al. (2009) não observaram diferença quanto ao IDS, IHS e QI, em policultivo de jundiá e carpa-húngara, e concluíram que esses parâmetros não são influenciados pela forma de cultivo, mas sim relacionados à composição da dieta.

Para os valores de QI e índice de gordura visceral (IGV), também não foram encontradas diferenças significativas entre as diferentes densidades e frequências testadas. Por sua vez, Lazzari et al. (2011)

Tabela 1. Parâmetros zootécnicos ${ }^{(1)}$ de juvenis de jundiá (Rhamdia quelen), criados em tanques-rede a diferentes densidades de estocagem (D) e frequências alimentares $(F)$.

\begin{tabular}{lccccccccccc}
\hline Tratamento $^{(2)}$ & PI $(\mathrm{g})$ & PF $(\mathrm{g})$ & GP $(\mathrm{g})$ & GMD $(\mathrm{g})$ & FC & TCE & U & SO & CT (cm) & RC (\%) & RF (\%) \\
\hline D-50, 50 jundiás por m ${ }^{3}$ & 56,91 & 99,19 & 42,75 & 0,70 & 0,89 & 0,93 & 0,57 & 0,90 & 22,34 & 81,11 & 28,20 \\
D-150, 150 jundiás por m ${ }^{3}$ & 58,04 & 105,47 & 47,70 & 0,79 & 0,86 & 0,99 & 0,51 & 0,93 & 23,15 & 81,11 & 28,50 \\
F-1, alimentados uma vez ao dia & 57,11 & 102,36 & 45,20 & 0,75 & 0,82 & 0,96 & 0,54 & 0,88 & 23,16 & 80,40 & 28,30 \\
F-2, alimentados duas vezes ao dia & 57,84 & 102,30 & 45,26 & 0,74 & 0,93 & 0,96 & 0,55 & 0,95 & 22,33 & 81,70 & 28,40 \\
\hline Desvio-padrão residual & 3,35 & 8,60 & 5,98 & 0,10 & 0,10 & 0,08 & 0,09 & 0,55 & 0,97 & 0,20 & 20,02 \\
\hline Efeito da densidade (D) & $\mathrm{ns}$ & $\mathrm{ns}$ & $\mathrm{ns}$ & $\mathrm{ns}$ & $\mathrm{ns}$ & $\mathrm{ns}$ & $\mathrm{ns}$ & $\mathrm{ns}$ & $\mathrm{ns}$ & $\mathrm{ns}$ & $\mathrm{ns}$ \\
Efeito da frequência (F) & $\mathrm{ns}$ & $\mathrm{ns}$ & $\mathrm{ns}$ & $\mathrm{ns}$ & $\mathrm{ns}$ & $\mathrm{ns}$ & $\mathrm{ns}$ & $\mathrm{ns}$ & $\mathrm{ns}$ & $\mathrm{ns}$ & $\mathrm{ns}$ \\
Interação D $\times$ F & $\mathrm{ns}$ & $\mathrm{ns}$ & $\mathrm{ns}$ & $\mathrm{ns}$ & $\mathrm{ns}$ & $\mathrm{ns}$ & $\mathrm{ns}$ & $\mathrm{ns}$ & $\mathrm{ns}$ & $\mathrm{ns}$ & $\mathrm{ns}$ \\
\hline
\end{tabular}

(1)PI, massa inicial; PF, massa final; GP, ganho de massa; GMD, ganho médio diário; FC, fator de condição; TCE, taxa de crescimento específico (\% por dia); U, uniformidade; SO, sobrevivência; CT, comprimento total; RC, rendimento de carcaça; RF, rendimento de filé. ns Não significativo pelo teste de Duncan, a $5 \%$ de probabilidade.

Tabela 2. Índice digestivossomático (IDS), hepatossomático (IHS), índice de gordura visceral (IGV) e quociente intestinal (QI) de juvenis de jundiá (Rhamdia quelen), criados em tanques-rede a diferentes densidades de estocagem (D) e frequências alimentares $(\mathrm{F})^{(1)}$.

\begin{tabular}{|c|c|c|c|c|}
\hline Tratamento & Índice digestivo somático & Índice hepatossomático & Índice de gordura visceral & Quociente intestinal \\
\hline$\overline{\mathrm{D}-50,50 \text { jundiás por } \mathrm{m}^{3}}$ & $3,99 \pm 0,48$ & $3,20 \pm 0,54$ & $2,45 \pm 0,76$ & $2,05 \pm 0,19$ \\
\hline D-150, 150 jundiás por $\mathrm{m}^{3}$ & $4,07 \pm 0,24$ & $3,22 \pm 0,38$ & $2,23 \pm 0,71$ & $2,00 \pm 0,20$ \\
\hline F-1, alimentados uma vez ao dia & $4,09 \pm 0,44$ & $3,30 \pm 0,53$ & $2,21 \pm 0,88$ & $2,02 \pm 0,18$ \\
\hline F-2, alimentados duas vezes ao dia & $4,02 \pm 0,29$ & $3,16 \pm 0,38$ & $2,36 \pm 0,64$ & $2,01 \pm 0,21$ \\
\hline Efeito da densidade (D) & ns & ns & ns & ns \\
\hline Efeito da frequência $(F)$ & ns & ns & ns & ns \\
\hline Interação D x F & ns & ns & ns & ns \\
\hline
\end{tabular}

(1)ns Não significativo pelo teste de Duncan, a 5\% de probabilidade. 
ressaltaram que baixas densidades de estocagem podem levar ao maior acúmulo de gordura nos peixes, em consequência da menor competição por alimento.

As densidades e frequências alimentares não influenciaram a composição corporal de peixe inteiro (Tabela 3). Porém, os filés dos animais submetidos à menor densidade de estocagem, com frequência de arraçoamento de uma refeição diária, apresentaram maior teor de gordura do que aqueles mantidos à mesma densidade e recebendo duas refeições diárias (Tabela 4). Lazzari et al. (2011) encontraram resultados semelhantes para a composição do jundiá, ao testar as densidades de 4,1, 6,4, 8,6 e 10,8 $\mathrm{kg} \mathrm{m}^{-3}$, com diferença apenas quanto ao teor proteico do peixe inteiro.

Bittencourt et al. (2010) avaliaram a composição corporal de pacus (Piaractus mesopotamicus), cultivados a diferentes densidades de estocagem, em tanques-rede, e encontraram diferença apenas quanto à gordura, com menores valores obtidos às maiores densidades de estocagem.

A possível explicação para o menor teor lipídico, no tratamento à menor densidade e à maior frequência, pode ser vinculada à disputa por território (menor densidade), aliada à menor quantidade de alimento fornecida por refeição. Isso causou maior gasto energético e levou à menor deposição de gordura nos filés. Piaia \& Baldisseroto (2000), trabalharam com três densidades de estocagem para juvenis de jundiá - 114, 227 e 454 alevinos $\mathrm{m}^{-3}$ - e observaram que os animais cultivados a maiores densidades permanecem

Tabela 3. Composição centesimal (peixe inteiro) de juvenis de jundiás (Rhamdia quelen), cultivados em tanques-rede a diferentes densidades de estocagem (D) e frequências alimentares $(\mathrm{F})$.

\begin{tabular}{lcccc}
\hline Tratamento $^{(1)}$ & $\begin{array}{c}\text { Matéria } \\
\text { mineral (\%) }\end{array}$ & $\begin{array}{c}\text { Umidade } \\
(\%)\end{array}$ & $\begin{array}{c}\text { Gordura } \\
(\%)\end{array}$ & $\begin{array}{c}\text { Proteína } \\
(\%)\end{array}$ \\
\hline $\mathrm{D}-50$ & $3,19 \pm 0,36$ & $69,17 \pm 1,78$ & $10,19 \pm 1,74$ & $14,63 \pm 0,80$ \\
$\mathrm{D}-150$ & $3,30 \pm 0,45$ & $69,10 \pm 1,76$ & $10,66 \pm 1,61$ & $14,59 \pm 0,92$ \\
$\mathrm{~F}-1$ & $3,32 \pm 0,39$ & $69,17 \pm 1,53$ & $10,47 \pm 1,63$ & $14,63 \pm 0,95$ \\
$\mathrm{~F}-2$ & $3,17 \pm 0,41$ & $69,10 \pm 1,74$ & $10,37 \pm 1,75$ & $14,60 \pm 0,76$ \\
\hline $\mathrm{D}$ & $\mathrm{ns}$ & $\mathrm{ns}$ & $\mathrm{ns}$ & $\mathrm{ns}$ \\
$\mathrm{F}$ & $\mathrm{ns}$ & $\mathrm{ns}$ & $\mathrm{ns}$ & $\mathrm{ns}$ \\
Interação D $\times \mathrm{F}$ & $\mathrm{ns}$ & $\mathrm{ns}$ & $\mathrm{ns}$ & $\mathrm{ns}$ \\
\hline
\end{tabular}

(1) $\mathrm{D}-50,50$ jundiás por $\mathrm{m}^{3}$; $\mathrm{D}-150,150$ jundiás por $\mathrm{m}^{3} ; \mathrm{F}-1$, alimentados uma vez ao dia; $\mathrm{F}-2$, alimentados duas vezes ao dia; $\mathrm{D}$, efeito da densidade;

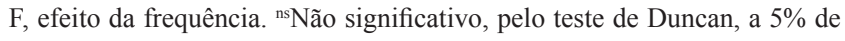
probabilidade. em grupos e formam cardumes na captura por alimento, o que não acontece com os indivíduos cultivados à menor densidade, que formam territórios distintos e individuais, geram disputas e, consequentemente, maior gasto energético.

Embora os lipídios tenham funções metabólicas essenciais para o metabolismo dos peixes (Fujimoto et al., 2007), quando estes atingem massa própria para a comercialização, a deposição de gordura pode ser indesejável, uma vez que tende a afetar as características organolépticas do produto. Assim, deve-se manter um nível de deposição lipídica que não interfira na qualidade sensorial do pescado (Meurer et al., 2003).

Não foram observadas diferenças significativas para os níveis de glicose, às densidades e frequências alimentares testadas (Tabela 5), o que mostra que as variações de manejo preconizadas não foram extremas para o cultivo do jundiá. Os níveis de colesterol, urato e triglicerídeos sanguíneos também não apresentaram diferença entre as densidades e frequências alimentares avaliadas. A avaliação dos parâmetros sanguíneos em peixes é de extrema importância, pois alterações nestes parâmetros podem indicar mobilização de reservas ou também podem ser indicativo de algum distúrbio fisiológico (Brandão et al., 2004; Chagas et al., 2007). $\mathrm{O}$ aumento da glicose sanguínea pode indicar estado de estresse nos peixes (Barcellos et al., 2000) e, em densidades de estocagem extremas, pode haver aumento nos níveis sanguíneos deste parâmetro (Brandão et al., 2004). Montero et al. (1999), verificaram aumento nos níveis de glicose e proteína no sangue de Sparus aurata mantidos a altas densidades $\left(40,8 \mathrm{~kg} \mathrm{~m}^{-3}\right)$.

Tabela 4. Composição centesimal dos filés de jundiá (Rhamdia quelen), cultivados em tanques-rede a diferentes densidades de estocagem e frequências alimentares ${ }^{(1)}$.

\begin{tabular}{lcccc}
\hline Composição & \multicolumn{4}{c}{ Tratamento $^{(2)}$} \\
\cline { 2 - 5 } & $50 / 1$ & $50 / 2$ & $150 / 1$ & $150 / 2$ \\
\hline Cinzas (\%) & $1,18 \pm 0,11$ & $1,33 \pm 0,31$ & $1,21 \pm 0,19$ & $1,19 \pm 0,14$ \\
Umidade (\%) & $70,04 \pm 1,31$ & $71,45 \pm 2,56$ & $70,89 \pm 1,02$ & $69,57 \pm 1,822$ \\
Gordura (\%) & $10,48 \pm 1,43 \mathrm{a}$ & $8,10 \pm 0,74 \mathrm{~b}$ & $9,14 \pm 1,86 \mathrm{ab}$ & $10,07 \pm 2,24 \mathrm{ab}$ \\
Proteína (\%) & $17,80 \pm 1,74$ & $16,11 \pm 1,43$ & $17,05 \pm 1,08$ & $17,43 \pm 1,24$ \\
\hline
\end{tabular}

${ }^{(1)}$ Médias seguidas de letras iguais, nas linhas, não diferem pelo teste de Duncan, a $5 \%$ de probabilidade. ${ }^{(2)} 50 / 1,50$ jundiás por $\mathrm{m}^{3}$, alimentados uma vez ao dia; 50/2, 50 jundiás por $\mathrm{m}^{3}$, alimentados duas vezes ao dia; $150 / 1$, 150 jundiás por $\mathrm{m}^{3}$, alimentados uma vez ao dia; $150 / 2,150$ jundiás por $\mathrm{m}^{3}$, alimentados duas vezes ao dia.

Pesq. agropec. bras., Brasília, v.48, n.8, p.871-877, ago. 2013 DOI: $10.1590 / \mathrm{S} 0100-204 X 2013000800009$ 
Tabela 5. Parâmetros sanguíneos de juvenis de jundiá (Rhamdia quelen), criados em tanques-rede a diferentes densidades de estocagem (D) e frequências alimentares (F).

\begin{tabular}{lccccc}
\hline Fatores & $\begin{array}{c}\text { Colesterol } \\
\left(\mathrm{mg} \mathrm{dL}^{-1}\right)\end{array}$ & $\begin{array}{c}\text { Glicose } \\
\left(\mathrm{mg} \mathrm{dL}^{-1}\right)\end{array}$ & $\begin{array}{c}\text { Proteína } \\
\left(\mathrm{g} \mathrm{dL}^{-1}\right)\end{array}$ & $\begin{array}{c}\text { Urato } \\
\left(\mathrm{g} \mathrm{dL}^{-1}\right)\end{array}$ & $\begin{array}{c}\text { Triglicerídeos } \\
\left(\mathrm{mg} \mathrm{dL}^{-1}\right)\end{array}$ \\
\hline D-50, 50 jundiás por $\mathrm{m}^{3}$ & $203,07 \pm 66,98$ & $34,14 \pm 6,10$ & $2,97 \pm 0,37 \mathrm{a}$ & $0,71 \pm 0,53$ & $420,33 \pm 239,88$ \\
D-150, 150 jundiás por $\mathrm{m}^{3}$ & $190,16 \pm 59,97$ & $32,50 \pm 8,46$ & $2,51 \pm 0,44 \mathrm{~b}$ & $0,70 \pm 0,37$ & $308,45 \pm 250,04$ \\
F-1, alimentados uma vez ao dia & $177,94 \pm 51,04$ & $35,22 \pm 8,92$ & $2,61 \pm 0,49$ & $0,61 \pm 0,26$ & $400,10 \pm 255,95$ \\
F-2, alimentados duas vezes ao dia & $215,28 \pm 69,34$ & $31,42 \pm 4,92$ & $2,86 \pm 0,41$ & $0,79 \pm 0,56$ & $328,69 \pm 242,07$ \\
\hline Efeito da densidade (D) & $\mathrm{ns}$ & $\mathrm{ns}$ & $*$ & $\mathrm{~ns}$ & $\mathrm{~ns}$ \\
Efeito da frequência (F) & $\mathrm{ns}$ & $\mathrm{ns}$ & $\mathrm{ns}$ & $\mathrm{ns}$ & $\mathrm{ns}$ \\
Interação D x F & $\mathrm{ns}$ & $\mathrm{ns}$ & $\mathrm{ns}$ & $\mathrm{ns}$ & $\mathrm{ns}$ \\
\hline
\end{tabular}

*Significativo pelo teste de Duncan, a 5\% de probabilidade. ${ }^{\text {ns }}$ Não significativo.

$\mathrm{Na}$ avaliação das proteínas totais sanguíneas, os níveis no plasma diferiram entre as densidades de 50 e 150 peixes $\mathrm{m}^{-3}$, com maiores resultados na densidade de 50 peixes $\mathrm{m}^{-3}$. Isto pode estar relacionado à menor disputa por alimento no tratamento à densidade mais baixa. Não se observou diferença, nos valores deste parâmetro, quanto às frequências alimentares. Chagas et al. (2007) também não encontraram diferenças nos valores de proteínas totais sanguíneas $\left(2,45 \mathrm{~g} \mathrm{dL}^{-1}\right)$, quando avaliaram o efeito da taxa de alimentação sobre a produtividade de tambaqui, em tanques-rede.

\section{Conclusão}

A densidade de 150 peixes por $\mathrm{m}^{3}$ e a frequência de arraçoamento uma vez ao dia não afetam o desempenho zootécnico dos jundiás (Rhamdia quelen).

\section{Agradecimentos}

Ao Conselho Nacional de Desenvolvimento Científico e Tecnológico (CNPq) e Ministério da Pesca e Aquicultura, por auxílio financeiro e concessão de bolsas.

\section{Referências}

ADAD, J.M.T. Controle químico de qualidade da água. Rio de Janeiro: Guanabara, 1982. 203p.

ALMEIDA, S.C.A. de; NUÑER, A.P. de O. Crescimento de Pimelodus maculatus (Actinopterygii, Pimelodidae) estocados em diferentes densidades em tanques-rede. Revista Biotemas, v.22, p.113-119, 2009.

BALDISSEROTTO, B.; GOMES, L. de C. Espécies nativas para piscicultura no Brasil. Santa Maria: Ed. UFSM, 2005. 303p.
BARCELlOS, L.J.G.; SOUZA, S.M.G. de; WOEHL, V.M. Estresse em peixes: fisiologia da resposta ao estresse, causas e consequências (revisão). Boletim do Instituto da Pesca, v.26, p.99-111, 2000.

BITTENCOURT, F.; FEIDEN, A.; SIGNOR, A.A.; BOSCOLO, W.R.; LORENZ, E.K.; MALUF, M.L.F. Densidade de estocagem e parâmetros eritrocitários de pacus criados em tanques-rede. Revista Brasileira de Zootecnia, v.39, p.2323-2329, 2010. DOI: 10.1590/S1516-35982010001100002.

BLIGH, E.G.; DYER, W.J. A rapid method of total lipid extraction and purification. Canadian Journal of Biochemistry and Physiology, v.37, p.911-917, 1959. DOI: 10.1139/059-099.

BRANDÃO, F.R.; GOMES, L. de C.; CHAGAS, E.C.; ARAÚJO, L.D. de. Densidade de estocagem de juvenis de tambaqui durante a recria em tanques-rede. Pesquisa Agropecuária Brasileira, v.39, p.357-362, 2004. DOI: 10.1590/ S0100-204X2004000400009.

BRASIL. Ministério da Pesca e Aquicultura. Produção pesqueira e aquícola, estatísticas 2003-2009. 2010. Disponível em: <http://www.mpa.gov.br/\#info-estatistica/ estatistica-da-pesca-e-aquicultura $>$. Acesso em: 20 mar. 2011.

CANTON, R.; WEINGARTNER, M.; FRACALOSSI, D.M.; ZANIBONI FILHO, E. Influência da frequência alimentar no desempenho de juvenis de jundiá. Revista Brasileira de Zootecnia, v.36, p.749-753, 2007. DOI: 10.1590/ S1516-35982007000400001.

CARNEIRO, P.C.F.; MIKOS, J.D. Frequência alimentar e crescimento de alevinos de jundiá, Rhamdia quelen. Ciência Rural, v.35, p.187-191, 2005. DOI: 10.1590/S0103-84782005000100030.

CHAGAS, E.C.; GOMES, L. de C.; MARTINS JÚNIOR, H.; ROUBACH, R. Produtividade de tambaqui criado em tanque-rede com diferentes taxas de alimentação. Ciência Rural, v.37, p.1109-1115, 2007. DOI: 10.1590/S0103-84782007000400031.

CORRÊIA, V.; RADÜNZ NETO, J.; LAZZARI, R.; VEIVERBERG, C.A.; BERGAMIN, G.T.; PEDRON, F. de A.; FERREIRA, C.C.; EMANUELLI, T.; RIBEIRO, C.P. Crescimento de jundiá e carpa húngara criados em sistema de recirculação de água. Ciência Rural, v.39, p.1533-1539, 2009. DOI: 10.1590/ S0103-84782009005000070. 
CORRÊIA, V.; RADÜNZ NETO, J.; ROSSATO, S.; MASCHIO, D.; MARTINELLI, S.G. Efeito da densidade de estocagem e a resposta de estresse no policultivo de jundiá (Rhamdia quelen) e carpa húngara (Cyprinus carpio). Revista da FZVA, v.17, p.170-185, 2010.

CUNHA, M.A. da; ZEPPENFELD, C.C.; GARCIA, L. de O.; LORO, V.L.; FONSECA, M.B. da; EMANUELLI, T.; VEECK, A.P. de L.; COPATTI, C.E.; BALDISSEROTTO, B. Anesthesia of silver catfish with eugenol: time of induction, cortisol response and sensory analysis of fillet. Ciência Rural, v.10, p 2107-2114, 2010. DOI: $10.1590 / \mathrm{S} 0103-84782010005000154$.

CUNNIFF, P. Official methods of analysis of AOAC International. $16^{\text {th }}$ ed. Washington: AOAC International, 1995. 1141p.

FRACALOSSI, D.M.; MEYER, G.; SANTAMARIA, F.M.; WEINGARTNER, M.; ZANIBONI FILHO, E. Desempenho do jundiá, Rhamdia quelen, e do dourado, Salminus brasiliensis, em viveiros de terra na Região Sul do Brasil. Acta Scientiarum. Animal Sciences, v.26, p.345-352, 2004.

FUJIMOTO, R.Y.; CASTRO, M.P. de; HONORATO, C.A.; MORAES, F.R. de. Composição corporal e eficiência de utilização de nutrientes por pacus alimentados com ração suplementada com cromo trivalente. Pesquisa Agropecuária Brasileira, v.42, p.1763-1768, 2007. DOI: 10.1590/ S0100-204X2007001200014.

FURLANETO, F.P.B.; AYROZA, D.M.M.R.; AYROZA, L.M.S. Custo e rentabilidade da produção de tilápia (Oreochromis spp.) em tanque-rede no Médio Paranapanema, Estado de São Paulo, safra 2004/05. Informações Econômicas, v.36, p.63-69, 2006.

FURUYA, W.M.; SOUZA, S.R. de; FURUYA, V.R.B.; HAYASHI, C.; RIBEIRO, R.P. Dietas peletizada e extrusada para machos revertidos de tilápias do Nilo (Oreochromis niloticus L.), na fase de terminação. Ciência Rural, v.28, p.483-487, 1998. DOI: 10.1590/ S0103-84781998000300022.

JOBLING, M. Fish bioenergetics. London: Chapman and Hall, 1994. 309p.

LAZZARI, R.; RADÜNZNETO, J.; CORRÊIA,V.; VEIVERBERG, C.A.; BERGAMIN, G.T.; EMANUELLI, T.; RIBEIRO, C.P. Densidade de estocagem no crescimento, composição e perfil lipídico corporal do jundiá. Ciência Rural, v.41, p.712-718, 2011. DOI: $10.1590 / \mathrm{S} 0103-84782011000400027$.

LOSEKANN, M.E.; RADÜNZ NETO, J.; EMANUELLI, T.; PEDRON, F. de A.; LAZZARI, R.; BERGAMIN, G.T.; CORRÊIA, V.; SIMÕES, R.S. Alimentação do jundiá com dietas contendo óleos de arroz, canola ou soja. Ciência Rural, v.38, p.225-230, 2008. DOI: $10.1590 / \mathrm{S} 0103-84782008000100036$.

MEURER, F.; HAYASHI, C.; BOSCOLO, W.R. Digestibilidade aparente de alguns alimentos protéicos pela tilápia do Nilo (Oreochromis niloticus). Revista Brasileira de Zootecnia, v.32, p.1801-1809, 2003. DOI: 10.1590/ S1516-35982003000800001.

MONTERO, D.; IZQUIERDO, M.S.; TORT, L.; ROBAINA, L.; VERGARA, J.M. High stocking density produces crowding stress altering some physiological and biochemical parameters in gilthead seabream, Sparus aurata, juveniles. Fish Physiology and Biochemistry, v.20, p.53-60, 1999. DOI: 10.1023/A:1007719928905.

PEDRON, F. de A.; RADÜNZ NETO, J.; EMANUELLI, T.; SILVA, L.P. da; LAZZARI, R.; CORRÊIA, V.; BERGAMIN, G.T.; VEIVERBERG, C.A. Cultivo de jundiás alimentados com dietas com casca de soja ou de algodão. Pesquisa Agropecuária Brasileira, v.43, p.93-98, 2008. DOI: 10.1590/ S0100-204X2008000100012.

PIAIA, R.; BALDISSEROTTO, B. Densidade de estocagem e crescimento de alevinos de jundiá Rhamdia quelen (Quoy \& Gaimard, 1824). Ciência Rural, v.30, p.509-513, 2000. DOI: 10.1590/S0103-84782000000300024.

REIDEL, A.; ROMAGOSA, E.; FEIDEN, A.; BOSCOLO, W.R.; COLDEBELlA, A.; SIGNOR, A.A. Rendimento corporal e composição química de jundiás alimentados com diferentes níveis de proteína e energia na dieta, criados em tanques-rede. Revista Brasileira de Zootecnia, v.39, p.233-240, 2010. DOI: 10.1590/ S1516-35982010000200001.

SALARO, A.L.; LUZ, R.K.; NOGUEIRA, G.C.C. de B.; REIS, A.; SAKABE, R.; LAMBERTUCCI, D.M. Diferentes densidades de estocagem na produção de alevinos de trairão (Hoplias cf. lacerdae). Revista Brasileira de Zootecnia, v.32, p.1033-1036, 2003. DOI: 10.1590/S1516-35982003000500001.

VEIVERBERG, C.A.; BERGAMIN, G.T.; RADÜNZ NETO, J.; LAZZARI, R.; ROSSATO, S.; SUTILI, F.J.; FERREIRA, C.C. Farelo de soja como substituto à farinha de carne e ossos em dietas para juvenis de carpa capim (Ctenopharyngodon idella). Boletim do Instituto da Pesca, v.34, p.463-472, 2008.

VERDOUW, H.; VAN ECHTELD, C.J.A.; DEKKERS, E.M.J. Ammonia determination based on indophenol formation with sodium salicylate. Water Research, v.12, p.399-402, 1997. DOI: 10.1016/0043-1354(78)90107-0.

Recebido em 31 de julho de 2011 e aprovado em 2 de julho de 2013 